\title{
Prices of pork cuts : Retrospective analysis and prediction attempts
}

\author{
M. RIEU et A. VIGNE \\ Institut Technique du Porc, 34, bd de la Gare, 31500 Toulouse
}

The impact of prices of pork cuts becomes more and more important for the economy of the whole pig channel. It affects the income of farmers and that of processing industries. Like the carcass price, and often more than it, the price of each cut is extremely variable.

On the basis of methods applied to pig carcass prices, the authors studied the variation of cut prices in France over the last two decades : decomposition into trend, cycle, season and residue, then analysis of theses components. Achieving periodically a one-year estimation of carcass prices, they attempted to deduce simply an estimation of cut prices.

The analysis showed that the cycle has an effect on all cut prices. Its characteristics, period, phase, magnitude are sometimes close (loin, ham, neck), sometimes remote (belly, backfat, flare fat) from those of the carcass.

Compared to that of the carcass over the twenty years, the trend may display some variations (loin, ham) or more or less marked up- and downgradings (belly, neck, fat pieces).

For prediction, the cycle or trend differences between cut and carcass are obstacles to high quality results. The latter are satisfactory for loin, ham, neck, poor for belly and fat pieces.

\section{Factors affecting present trends in pig slaughtering and processing industries}

\author{
C. RENAULT \\ Association Nutrition Demain, 61 A, rue d'Avignon, M.I.N., \\ Fruileg 682, 94574 Rungis
}

The purpose of the present study is to define the factors affecting the structural changes in the pig slaughtering, cutting and processing sector. This work is based on the study of 28 slaughtering and cutting industries and 30 processing industries.

Results show that beside size and specialization criteria, two elements are important for the development of pig industries : their progress in the transformation processes and the relevance of their commercial policy.

\section{Influence of several favourable factors on pork consumption}

\author{
P. MAINSANT \\ Institut National de la Recherche Agronomique, \\ Laboratoire de Recherches Economiques et Sociales, 3, rue du Caducée, B.P. 333, \\ 94153 Rungis Cedex
}

During the post-war period, the increase of income has led to a higher consumption of pork and beef. From the sixties, because of differences in retail prices, pork has progressed faster than 
beef. This trend was observed in all E.E.C. countries. Since 1980, meat consumption has become stabilized.

Analysis of the last ten years through the Secodip panel shows that the price effect is still in favour of pork consumption. The reduction of retail prices results from advances in productivity. Moreover, these reductions are better transferred to the consumer by modern retailers than by traditional distribution especially through seasonal special offers. These two advantages are rather stable in the mean term.

As regards processed products, consumption is more variable. Cooked and raw ham have rapidly progressed even recently thanks to a higher quality, the distribution in large and medium supermarkets, the ready-to-use character and marketing campaigns of manufacturers. By contrast, dry sausages and patties stagnate whereas fresh sausages, "terrines ", "pâtés en croûte " and catering products progress.

On the overall, the pig channel seems to follow the present market trend, since through improvements of its production and industry it progresses in terms of price, quality and variety of processed products.

\title{
PATHOLOGIE - PATHOLOGY
}

\section{Main viral and contagious diseases of the pig: Epidemiological situation in France and Europe}

\author{
Ph. VANNIER ${ }^{(1)}$, P. LE FOLL ${ }^{(2)}$ \\ (1) Station de Pathologie Porcine, B.P. 9, 22440 Ploufragan \\ (2) Institut Technique du Porc, 34, boulevard de la Gare, 31500 Toulouse
}

The sanitary state of French pig herds was compared to that of the neighbouring countries in Europe. The story of the Aujeszky's disease can be divided into three periods. From 1984 control measures have been applied according to regional conditions established from a preliminary epidemiological inquiry. In four French regions, the efficiency of the applied measures can be estimated. Except in two "departements", the eradication programme can be considered as successful with a moderate average cost ( $30 \mathrm{FF}$ per sow and per year). In two other European countries, an eradication scheme was launched: Denmark and Great-Britain. In these countries, it seems that the A.D. has been eliminated but, in Great-Britain farmers would have to pay 30 pence per sold pig until mid-1989, i.e. for 6 years. In the other countries, the situation is more confused and difficult to evaluate. In Belgium and the Netherlands, all breeders are systematically vaccinated.

Although an eradication programme for Classical Swine Fever (C.S.F.) has been launched in 1980 in the E.E.C., the prevalence of the disease is variable from one country to another.

Denmark, Great-Britain and Ireland are officially free from C.S.F. In France, outbreaks recorded the last two years are due to the import of infected animals or to the use of swill in the eastern part of the country. No vaccination has been performed since 1983. After a severe epizooty of C.S.F. between 1983 and 1985 in Belgium, the Netherlands and Germany, the number of outbreaks sharply decreased in these countries. Nevertheless the proportion of vaccinated pigs remains high nowadays.

African Swine Fever (A.S.F.) exists in enzootic state in Spain, Portugal and Sardinia. The other E.E.C. countries are permanently threatened. A.S.F. outbreaks were reported in 1985 in Belgium and in 1986 in the Netherlands. The initial outbreaks were provoked by the intake of infected swill.

Finally, over the last five years, except in Belgium, France, Great-Britain and Switzerland, no European country remained free from foot and mouth disease, but generally, except in Italy, the number of outbreaks was small and the infection was rapidly controlled. 